\title{
Probiotics and infective endocarditis in patients with hereditary hemorrhagic telangiectasia: a clinical case and a review of the literature
}

\author{
Evangelo Boumis* (D), Alessandro Capone, Vincenzo Galati, Carolina Venditti and Nicola Petrosillo
}

\begin{abstract}
Background: In the last decades, probiotics have been widely used as food supplements because of their putative beneficial health effects. They are generally considered safe but rare reports of serious infections caused by bacteria included in the definition of probiotics raise concerns on their potential pathogenic role in patients with particular predisposing factors. Patients with hereditary hemorrhagic telangiectasia $(H H T)$ are exposed to infections because of telangiectasias and arteriovenous malformations (AVMs). We describe what is, to our knowledge, the first case of infective endocarditis (IE) caused by Lactobacillus rhamnosus in a patient with HHT. A systematic review of the relevant medical literature is presented.
\end{abstract}

Case presentation: A patient with HHT and an aortic bioprosthesis was admitted because of prolonged fever not responding to antibiotics. The patient had a history of repeated serious infections with hospitalizations and prolonged use of antibiotics, and used to assume large amounts of different commercial products containing probiotics. Weeks before the onset of symptoms the patient had been treated with nasal packings and with surgical closure of a nasal bleeding site because of recurrent epistaxis. A diagnosis of IE of the aortic bioprosthesis was made. All blood coltures were positive for L. rhamnosus. The patients responded to a cycle of 6 weeks of amoxicillin/clavulanate plus gentamicin. A systematic review of IE linked to consumption of probiotics, and of infective endocarditis in patients with HHT was conducted. 10 cases of IE linked to probiotics consumption and 6 cases of IE in patients with HHT were found.

Conclusions: Consumption of probiotics can pose a risk of serious infections in patients with particular predisposing factors. Patients with HHT can be considered at risk because of their predisposition to infections. Prophylaxis with antibiotics before nasal packings in patients with HHT can be considered.

Keywords: Probiotics, Lactobacillus rhamnosus, Infective endocarditis, Hereditary hemorrhagic telangiectasia

\section{Background}

Probiotics are described as live microbial feed supplements that beneficially affect the host animal by improving its microbial balance [1]. Many microorganisms are considered probiotics, among these bacterial species of Bifidobacterium, Lactobacillus, Streptococcus, Enterococcus and yeasts like Saccharomyces. Many of these microorganisms constitute part of the normal human flora.

\footnotetext{
* Correspondence: eboumis@gmail.com

National Institute for Infectious Diseases "Lazzaro Spallanzani" IRCCS, Rome, Italy
}

(c) The Author(s). 2018 Open Access This article is distributed under the terms of the Creative Commons Attribution 4.0 International License (http://creativecommons.org/licenses/by/4.0/), which permits unrestricted use, distribution, and reproduction in any medium, provided you give appropriate credit to the original author(s) and the source, provide a link to the Creative Commons license, and indicate if changes were made. The Creative Commons Public Domain Dedication waiver (http://creativecommons.org/publicdomain/zero/1.0/) applies to the data made available in this article, unless otherwise stated.

Lactobacilli for example are normally present in the oral cavity, ileum and colon and are the dominant microorganisms in the vagina [2]; these are also the most widely represented microorganisms in probiotics.

The use of probiotics is widespread. Traditionally, in many countries they are consumed as fermented dairy food products like yogurt. Moreover, in the last two decades their use as food supplements has greatly increased because of their putative beneficial health effects (https://www.naturalproductsinsider.com/ articles/2016/09/the-new-market-profile-of-probiotics- 
consumption.aspx) [3]. Their use has also increased in the healthcare setting, especially among patients with impaired intestinal integrity and chronic conditions or immunosuppression, like diabetes mellitus, chronic renal failure, HIV infection, cirrhosis, neoplasia and organ transplant [4-7].

Probiotics are generally considered safe. Rare infections involving lactobacilli or bifidobacteria have been reported; however, the widespread use of probiotics in the general population did not seem to determine an increase of infections [8].

Hereditary hemorrhagic telangiectasia (HHT) or Rendu-Osler-Weber disease is an autosomal dominant disorder characterized by cutaneous telangiectasia, recurrent epistaxis and visceral arteriovenous malformations (AVMs) of the lungs, gastrointestinal tract, liver, and brain. Because AVMs allow the direct communication between pulmonary and systemic circulation, patients with HHT are at higher risk for severe infections like cerebral abscesses, septicemia, arthritis and osteomyelitis; among extracerebral infections, endocarditis is very seldom reported. [9].

Hereby we report a case of infective endocarditis caused by Lactobacillus rhamnosus in a patient with HHT who was also a heavy consumer of probiotics, and a review of the literature on similar cases. To our knowledge, this was the first case of infective endocarditis caused by a probiotic strain in a patient with HHT.

\section{Case presentation}

A patient aged $>65$ years, affected by HHT, was admitted in March, 2017 to our Institute because of prolonged fever not responding to antibiotics. The patient was the first of 4 siblings; two of them were also affected by HHT. In 2010 after a screening visit in a reference center for HHT a screening for AVMs of the liver, lung and brain was made with negative results. Apart from repeated episodes of epistaxis, often requiring specialist ear, nose and throat (ENT) intervention, nasal packings and laser coagulation, the patient's medical history was remarkable for repeated infections that required hospitalization during the years. In 2011 the patient had a spondylodiscitis with bacteremia caused by Streptococcus mutans; in 2012 a febrile enteritis was diagnosed; in 2015 an aortic bioprosthesis was implanted because of aortic insufficiency. In July, 2016 the patient was hospitalized for septic shock caused by Staphylococcus aureus bacteremia. After this episode the patient was admitted to an intensive care unit and, subsequently, had a prolonged stay in a long term care facility; during this stay the patient had prolonged alterations in bowel movements with intermittent diarrhea and therefore started to take probiotics. The probiotics included seven different commercial products containing different types of bacteria and yeasts; three of these contained Lactobacillus rhamnosus, which was also the last probiotic used by the patient before the recent admission to our hospital. In November, 2016 the patient was transferred from the long term care facility to a medical ward because of bacteremia caused by Streptococcus mutans, which responded to a cycle of four weeks of IV antibiotics. During this hospitalization the patient continued to have alteration in bowel movements; repeated stool examinations for Clostridium difficile, Salmonella, Shigella and other enteric pathogens and a colonoscopy were negative. No obvious infectious foci were detected, and in December, 2016 the patient was discharged at home. Soon after discharge the patient was treated for recurrent epistaxis by an ENT specialist who performed laser coagulation of a nasal bleeding site.

In March, 2017, before the admission to our hospital, the patient, who continued to take probiotics, was treated at home by his attending physician with oral ciprofloxacin followed by IM ceftriaxone for a week, because of episodes of fever and night sweats, without improvement of symptoms. After 6 days of antimicrobial treatment, fever and night sweats persisted together with generalized fatigue and malaise and the patient was admitted to our hospital.

At admission the patient was febrile $\left(37.9{ }^{\circ} \mathrm{C}\right)$ and asthenic; pulse rate was $87 \mathrm{bpm}$; blood pressure was 110/ $70 \mathrm{mmHg}$. Skin examination revealed diffuse telangiectasia of the head, trunk and arms but was negative for signs of embolic phenomena. Cardiac examination revealed a $3 /$ 6 diastolic murmur. $\mathrm{C}$ reactive protein was elevated at $7.46 \mathrm{mg} / \mathrm{dL}$ (normal upper value <1); erythrocyte sedimentation rate was $100 \mathrm{~mm} / \mathrm{h}$; white blood cells were $4860 / \mu \mathrm{l}$ (neutrophils $72.5 \%$, lymphocytes $16 \%$ ); hemoglobin was $9.0 \mathrm{~g} / \mathrm{dL}$ and platelets were $218,000 / \mu \mathrm{l}$; albumin was $3.2 \mathrm{~g} / \mathrm{dL}$; blood chemistry, prothrombin time and liver enzymes were within the normal range. Procalcitonin was $0.08 \mathrm{ng} / \mathrm{mL}$, within the normal values. Serology for Salmonella, Brucella, Coxiella burnetii, Mycoplasma, Legionella was negative. Urine culture was also negative.

After three days of interruption of all antibiotics, three sets of blood cultures were drawn. A transesophageal echocardiogram reported an 11-mm endocarditic vegetation on the cuspids of the biologic prosthesis. According to Duke's criteria [10], a diagnosis of infective endocarditis was made. Empiric treatment with ceftriaxone $2 \mathrm{~g} /$ day and gentamicin $3 \mathrm{mg} / \mathrm{kg} /$ day, both by IV route, was started. Five blood cultures yielded gram positive rods, that were identified as Lactobacillus rhamnosus. This organism was sensitive to amoxicillin/ clavulanate and gentamicin but resistant to ceftriaxone; it was also resistant to penicillin, vancomycin, meropenem, rifampin and trimethoprim/sulphamethoxazole (see Additional file 1). The treatment was then modified; 
the patient was administered IV amoxicillin/clavulanate $2.2 \mathrm{~g}$ q $8 \mathrm{~h}$ plus IV gentamicin $3 \mathrm{mg} / \mathrm{kg} /$ day.

The clinical course was unremarkable with progressive clinical improvement. The patient tolerated well antibiotic treatment. Renal functionality remained normal in spite of prolonged administration of gentamicin. Repeat blood cultures performed during hospitalization were negative. Imaging of chest, abdomen, brain and spine showed no signs of embolization. A transesophageal echocardiogram performed after 5 weeks of treatment showed disappearance of the vegetation and a functioning prosthesis. The patient was discharged after a total of 6 weeks of treatment in good clinical conditions. At 6-month follow-up the patient was still in good health with no clinical and laboratory signs of infection.

Case reports of infective endocarditis presumably linked to consumption of probiotics, and of infective endocarditis in patients with HHT, reported between 1 January 1997 and 31 August 2017 were identified through a Medline search. We used the search terms "endocarditis", "probiotics", "Lactobacillus", "hereditary hemorrhagic telangiectasia" and "Rendu-Osler", and limited the search to publications in English, French and Spanish. Additional cases were identified from the references of the retrieved articles.

\section{Methods}

\section{Systematic review of the literature}

We found a total of 10 cases of infective endocarditis apparently linked to previous use of probiotics. We retrieved only 6 cases of infective endocarditis in patients with HHT. The results are shown in Tables 1 and 2, respectively [11-26].

\section{Discussion}

Endocarditis caused by probiotics and in patients with HHT are both rare conditions and the case we present is likely the result of different pathogenetic mechanisms operating in a single patient.

In cases of sepsis or bacteremia caused by microorganisms considered probiotics, mechanisms like bacterial translocation from the gut or other alterations in the integrity of mucosal surfaces permitting passage of microbes in the bloodstream have been hypothesized. In a series of 89 episodes of bacteremia caused by lactobacilli, the majority of patients had severe underlying diseases, with a predominance of gastrointestinal or hepatic neoplasia, and the mortality at 1 year was high (69\%). In this series, L. rhamnosus was the most frequent isolate (53\%); no cases of endocarditis were found [27]. In a series of 73 endocarditis caused by lactobacilli, $63 \%$ of patients had an underlying structural heart disease and $12 \%$ suffered from previous episodes of endocarditis.
Dental procedures were reported in $47 \%$ of cases, but consumption of probiotics was reported only in 3 cases [28].

In our literature search of endocarditis associated with probiotics use, we noticed that all patients had infections caused by microorganisms belonging to the species Lactobacillus. The majority of patients were not immunosuppressed, but instead had predisposing factors for bacteremia, like alteration in gut mucosal integrity, colonoscopy or teeth extraction. It is well known that in such circumstances patient may develop bacteremia and endocarditis caused by commensal microorganisms like oral streptococci or saprophytic microorganisms of the gut. The preponderance of Lactobacillus among infections caused by probiotics is interesting. It has been shown that Lactobacillus rhamnosus strains isolated from endocarditis possess the ability to aggregate platelets, to bind collagen and fibrinogen, and to produce glycosidases and proteases [29]. In an experimental study using the rat model of experimental endocarditis aimed at assessing the potential pathogenicity of probiotic and clinical isolates of Lactobacillus rhamnosus and Lactobacillus paracasei, it has been showed that at least some of the probiotic strains of $L$. rhamnosus exibit a $90 \%$ infective dose $\left(\mathrm{ID}_{90}\right)$ that is comparable to that of clinical isolates of Lactobacillus. These probiotic strains share the same fluorescent amplified fragment length polymorphism (FAFLP) as the clinical isolates with the lowest $\mathrm{ID}_{90}$, although they can be differentiated from clinical isolates by pulsed-field gel electrophoresis (PFGE) fingerprinting [30]. Moreover, strains of L. rhamnosus isolated in endocarditis and other serious infections that were indistinguishable from strains of probiotic L. rhamnosus by PFGE have been described $[15,31]$. These results suggest that at least in some circumstances probiotic strains of $L$. rhamnosus can give rise to bacteremia and endocarditis. Lactobacilli are intrinsically resistant to vancomycin, and may be resistant to various other antibiotics [32], so the treatment of infections caused by these microorganisms can be difficult.

In a series of 85 blood isolates of Lactobacillus [33] all species demonstrated low minimum inhibitory concentrations (MICs) of imipenem, piperacillin-tazobactam, netilmicin and clindamycin and high MICs of vancomycin; instead, the MICs of benzilpenicillin, ampicillin, ceftriaxone and other cephalosporins varied widely among species and among different isolates of the same species. Among cephalosporins, ceftriaxone showed the highest MIC values for isolates of L. rhamnosus. Ampicillin, broad-spectrum cephalosporins and vancomycin are often used in empiric treatment of endocarditis, but they could prove ineffective in bloodstream infection caused by lactobacilli.

People with HHT are predisposed to infections, because of the presence of pulmonary, hepatic and gut 


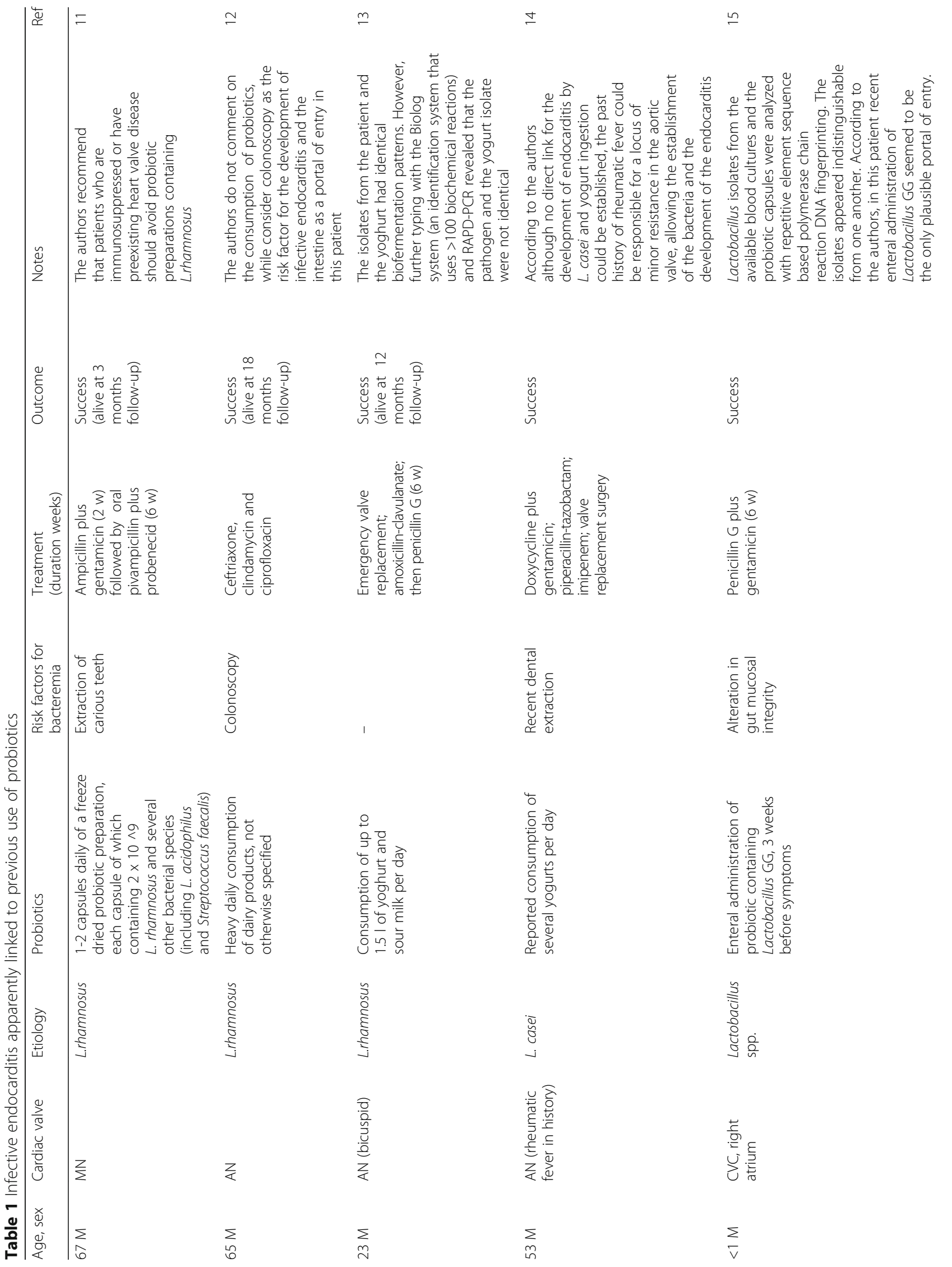




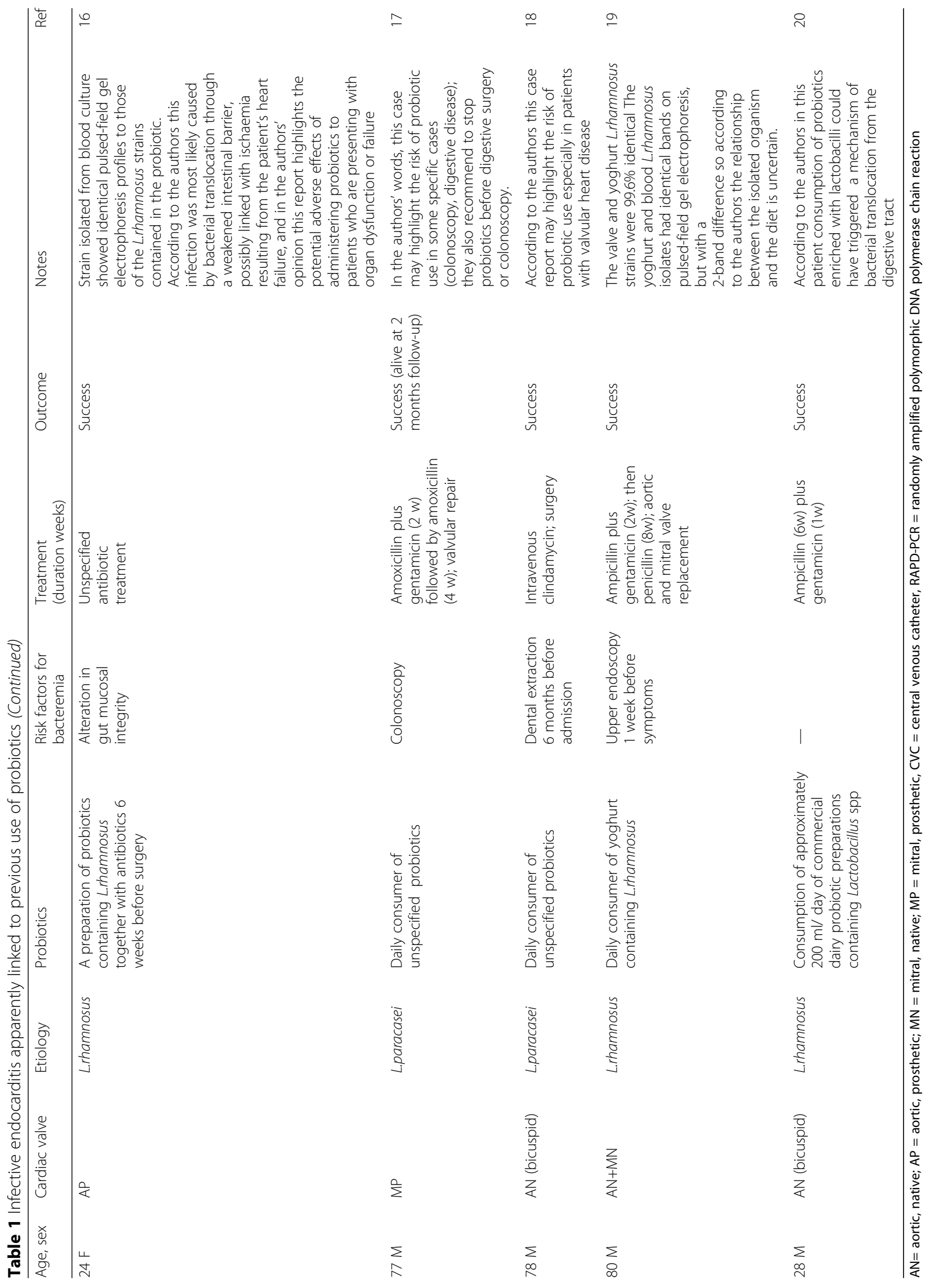




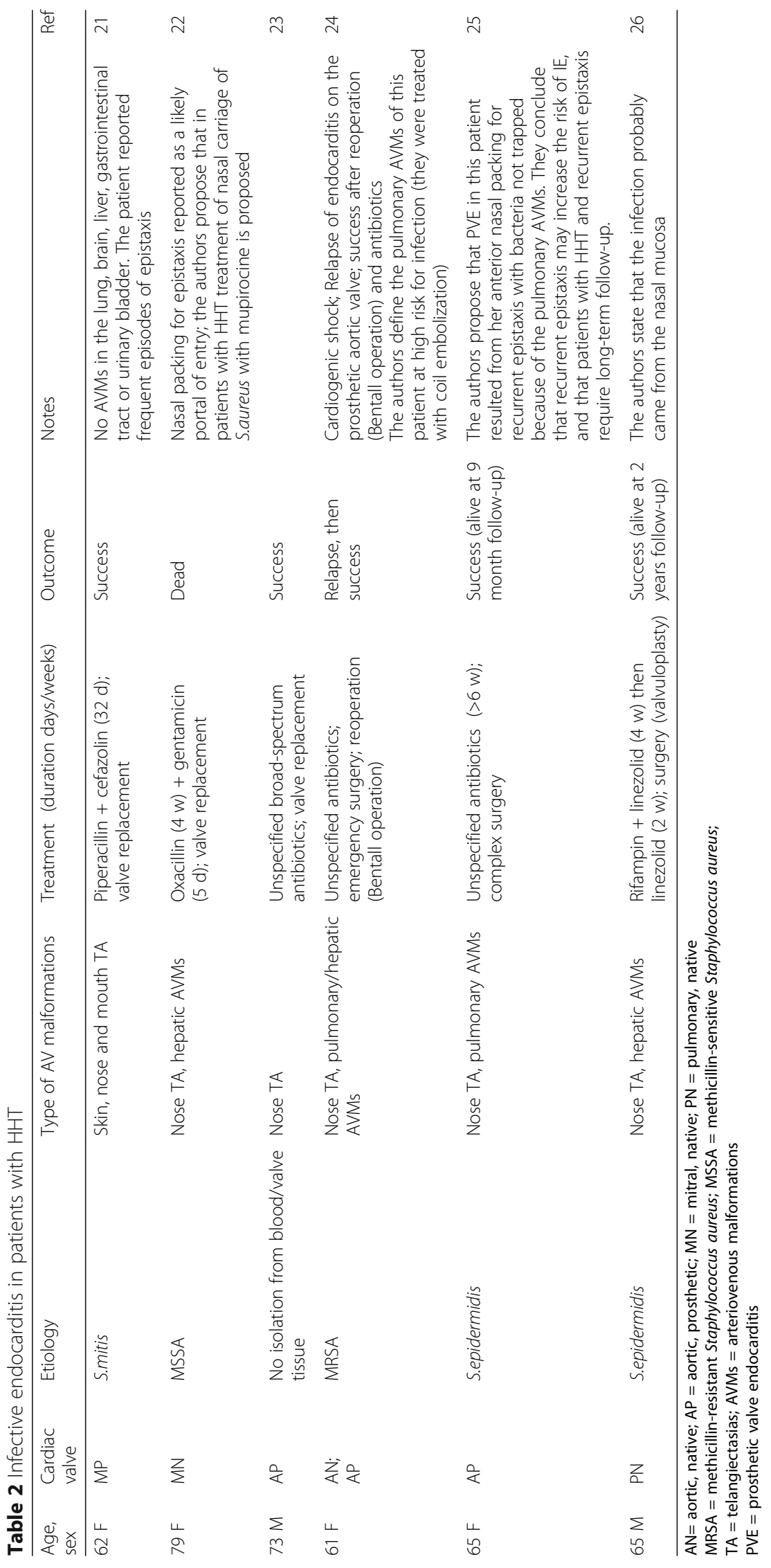


arterovenous malformation. The fragile mucosa of the nasal cavity is also considered a portal of entry for pathogenic microorganisms in these patients. The majority of infections reported in HHT patients are cerebral abscesses, however other infections like hepatic abscesses, bacteremia, septic arthritis, osteomyelitis, skin infections and infective endocarditis are also reported $[10,34]$. In our literature search, we found only 6 cases of infective endocarditis in patients with HHT, caused by coagulase positive and negative staphylococci and $S$. mitis. The mortality rate for these episodes was $16 \%$. The causative microorganisms were common commensal of the skin, nose, mouth, oropharyngeal tract and gut. The majority of the patients reported frequent epistaxis with nasal packing but no other risk factors for bacteremia. It has been hypothesized that nasal mucosa may be the portal of entry for microorganisms in case of nasal trauma associated with nasal packing; in other cases, proliferation of microorganisms like $S$. aureus was favored by the presence of foreign material in the nasal cavity $[35,36]$. It has been suggested that patients with HHT are considered at risk of bacteremia and endocarditis after dental procedures, and should be therefore treated with prophylactic antibiotics in these circumstances [37]; the same risk could be assumed for nasal procedures involving trauma to the nasal mucosa, and in these circumstances, antibiotic prophylaxis should probably be offered in these patients. It has also been suggested that these patient undergo nasal decontamination with mupirocin before nasal packing [38].

In the case of our patient, probably different factors have concurred to the occurrence of endocarditis. Although the patient's history of recurrent infections and bacteremia can raise the suspect of an immune depression, this was not apparently the case. The levels of lymphocytes, neutrophils, immunoglobulins and complement were normal during intervals free of disease. Human immunodeficiency virus (HIV) serology was negative; the patient had no signs of neoplasia and was not taking immunosuppressive drugs. In the patient's previous infective episodes, the isolates (Streptococcus mitis, Staphylococcus aureus) were typical pathogenic strains. Although the portal of entry of these bacteria was not clear, as apparently the patient had not an arteriovenous malformation in the lung, gut or brain, we hypothesize that the portal of entry was one of the numerous arteriovenous malformation in the nose or the oral cavity.

During the last infectious episode, the patient underwent a prolonged hospitalization in an intensive care unit and then in a long-term facility, received many courses of antibiotics and suffered from prolonged episodes of diarrhea; during this period the patient started to take large amounts of probiotics, many of them containing lactobacilli; therefore, it is plausible to assume that an imbalance in the bacterial flora of the gut and/or the oral cavity, and perhaps the nose, with a relative predominance of Lactobacillus could have occurred. We were not able to characterize the lactobacilli contained in the probiotics ingested by the patient, so we can only suppose that this strain of Lactobacillus rhamnosus was of dietary origin (we recognize that this is a limitation of our study). During the months before the last infectious episode, the patient had many episodes of prolonged epistaxis and underwent many traumatic nasal interventions such as nasal packing and cauterization of nasal telangiectasias. In patients with HHT, prolonged epistaxis is associated with extracerebral infections [9] and nasal instrumentation has been suggested as a possible risk factor for bacteremia [35]. Moreover, we cannot exclude other mechanisms and portals of entry of microbes in the bloodstream, such as microbial translocations of bacteria from the gut. Finally, the presence of a valvular aortic prosthesis was a clear predisposing factor for the development of infective endocarditis.

\section{Conclusions}

In conclusion, consumption of probiotics can pose a risk of serious infections in patients with particular predisposing factors to infections such as patients with HHT. These patients and their caregivers should be aware of the increased risk of infective endocarditis, especially during prolonged episodes of nasal bleeding and after nasal packing or other traumatic interventions in the nose. When these conditions are present, in our opinion antibiotic prophylaxis should be considered.

\section{Additional file}

Additional file 1: Microbiology. Description of methods used to identify the microorganism isolated from blood culture, both by standard microbiology culture and by genetic analysis; minimum inhibitory concentrations (MICs) of the antibiotics used for testing. (DOCX $11 \mathrm{~kb}$ )

\begin{abstract}
Abbreviations
AN: Aortic, native; AP: Aortic, prosthetic; AVMs: Arteriovenous malformations; CVC: Central venous catheter; ENT: Ear, nose and throat; FAFLP: Fluorescent amplified fragment length polymorphism; HHT: Hereditary hemorrhagic telangiectasia; HIV: Human immunodeficiency virus; ID $_{90}: 90 \%$ infective dose; IE: Infective endocarditis; IM: Intramuscular; IV: Intravenous; MICs: Minimum inhibitory concentrations; MN: Mitral, native; MP: Mitral, prosthetic;

MRSA: Methicillin-resistant Staphylococcus aureus; MSSA: Methicillin-sensitive Staphylococcus aureus; PFGE: Pulsed-field gel electrophoresis; PN: Pulmonary, native; PVE: Prosthetic valve endocarditis; RAPD-PCR: Randomly amplified polymorphic DNA polymerase chain reaction; TA: Telangiectasias
\end{abstract}

Acknowledgements

The authors thank Gabriella De Carli for editorial assistance.

Funding

None of the authors received funding for this study. 


\section{Availability of data and materials}

Not applicable (no datasets were generated or analyzed during the current study).

\section{Authors' contributions}

$E B, A C, V G$ and NP were involved in the clinical management of the case, performed the systematic review of the literature and contributed to the writing of the manuscript. CV analyzed blood samples for culture and performed genetic analysis of the isolate. All authors read and approved the final manuscript.

\section{Ethics approval and consent to participate}

Not applicable.

\section{Consent for publication}

The patient gave written consent to publish his case.

\section{Competing interests}

The authors declare that they have no competing interests.

\section{Publisher's Note}

Springer Nature remains neutral with regard to jurisdictional claims in published maps and institutional affiliations.

\section{Received: 5 October 2017 Accepted: 9 January 2018}

\section{Published online: 01 February 2018}

\section{References}

1. Fuller R. Probiotics in human medicine. Gut. 1991;32:439-42.

2. Walter J, Ley R. The human gut microbiome: ecology and recent evolutionary changes. Ann Rev Microbiol. 2011;65:411-29.

3. Nagpal R, Kumar A, Kumar M, et al. Probiotics, their health benefits and applications for developing healthier foods: a review. FEMS Microbiol Lett. 2012:334:1-15

4. Simkins J, Kaltsas A, Currie BP. Investigation of inpatient probiotic use at an academic medical center. Int J Infect Dis. 2013;17:e321-4.

5. Rayes N, Hansen S, Seehofer D, et al. Early enteral supply of fiber and lactobacilli versus conventional nutrition: a controlled trial in patients with major abdominal surgery. Nutrition. 2002;18:609-15.

6. Rayes N, Seehofer D, Hansen S, et al. Early enteral supply of lactobacillus and fiber versus selective bowel decontamination: a controlled trial in liver transplant recipients. Transplantation. 2002;74:123-7.

7. Redman MG, Ward EJ, Phillips RS. The efficacy and safety of probiotics in people with cancer: a systematic review. Ann Oncol. 2014;25:1919-29.

8. Salminen MK, Tynkkynen S, Rautelin $\mathrm{H}$, et al. Lactobacillus bacteremia during a rapid increase in probiotic use of Lactobacillus rhamnosus GG in Finland. Clin Infect Dis. 2002:35:1155-60.

9. Dupuis GS, Decullier E, Lesca G, et al. Hemorrhagic hereditary telangiectasia (Rendu-Osler disease) and infectious diseases: an underestimated association. Clin Infect Dis. 2007:44:841-5.

10. Li JS, Sexton DJ, Mick N, et al. Proposed modifications to the Duke criteria for the diagnosis of infective endocarditis. Clin Infect Dis. 2000:30:633-8,

11. Mackay AD, Taylor M, Kibbler CC, et al. Lactobacillus endocarditis caused by a probiotic organism. Clin Microbiol Infect. 1999;5:290-2.

12. Avlami A, Kordossis T, Vrizidis $\mathrm{N}$, et al. Lactobacillus rhamnosus endocarditis complicating colonoscopy. J Inf Secur. 2001:42:283-5.

13. Presterl E, Kneifel W, Mayer HK, et al. Endocarditis by lactobacillus rhamnosus due to yogurt ingestion? Scand J Infect Dis. 2001:33:710-4

14. Ze'-Ze' L, Tenreiro R, Duarte A, et al. Case of aortic endocarditis caused by Lactobacillus casei. J Med Microbiol. 2004;53:451-3.

15. Land MH, Rouster-Stevens $\mathrm{K}$, Woods $C R$, et al. Lactobacillus sepsis associated with probiotic therapy. Pediatrics. 2005:115:178-81.

16. Kochan P, Chmielarczyk A, Szymaniak L, et al. Lactobacillus rhamnosus administration causes sepsis in a cardiosurgical patient-is the time right to revise probiotic safety guidelines? Clin Microbiol Infect. 2011;17:1587-92.

17. Franko B, Vaillant $M$, Recule $C$, et al. Lactobacillus paracasei endocarditis in a consumer of probiotics. Med Mal Infect. 2013;43:170-3.

18. Kato K, Funabashi N, Takaoka H, et al. Lactobacillus paracasei endocarditis in a consumer of probiotics with advanced and severe bicuspid aortic valve stenosis complicated with diffuse left ventricular mid-layer fibrosis. Intern. J Cardiol. 2016:224:157-61.

19. Aaron JG, Sobieszczyk ME, Weiner SD. Lactobacillus rhamnosus endocarditis after upper endoscopy. Open Forum Infect Dis. 2017:4:1-3.

20. Noreña I, Cabrera-Marante O, Fernández-Ruiz M. Endocarditis due to lactobacillus rhamnosus in a patient with bicuspid aortic valve: potential role for the consumption of probiotics? Med Clin. 2017;149:181-2.

21. Ishikawa S, Oshima K, Aizaki M, et al. A second mitral valve replacement in a patient with hereditary hemorrhagic telangiectasia (Osler's disease). Jpn Heart J. 2004;45:885-8.

22. Bally C, Meyssonnier V, Bricaire F. Infections récidivantes à Staphylococcus aureus. Med Mal Infect. 2011;41:346-8.

23. Castiglioni A, Pozzoli A, Maisano F, et al. Endocarditis after transfemoral aortic valve implantation in a patient with Osler-weber-Rendu syndrome. Interact Cardiovasc Thorac Surg. 2012;15:553-4.

24. Morita $H, M D$, Kimura N, Yuri K, et al. Bentall operation for prosthetic valve endocarditis with hereditary hemorrhagic telangiectasia. Ann Thorac Cardiovasc Surg. 2014;20 Suppl:702-704

25. Nakamura $Y$, Shikata F, Ryugo $M$, et al. Redo cardiac surgery for active prosthetic valve endocarditis associated with hereditary hemorrhagic telangiectasia: report of a case. Surg Today. 2014;44:2378-81.

26. Konishi Y, Sakon Y, Nakamura K, et al. Pulmonary Valvuloplasty by autologous pericardium in a patient with Activelnfectious endocarditis and Osler's disease. J Heart Valve Dis. 2015;24:383-5.

27. Salminen MK, Rautelin H, Tynkkynen S, et al. Lactobacillus bacteremia, clinical significance, and patient outcome, with special focus on probiotic L. Rhamnosus GG. Clin Infect Dis. 2004;38:62-9.

28. Cannon JP, Lee TA, Bolanos JT, et al. Pathogenic relevance of lactobacillus: a retrospective review of over 200 cases. Eur J Clin Microbiol Infect Dis. 2005;24:31-40

29. Oakey HJ, Harty DW, Knox KW. Enzyme production by lactobacilli and the potential link with infective endocarditis. J Appl Bacteriol. 1995;78:142-8.

30. Vankerckhoven V, Moreillon P, Piu S, et al. Infectivity of lactobacillus rhamnosus and lactobacillus paracasei isolates in a rat model of experimental endocarditis. J Med Microbiol. 2007;56:1017-24.

31. Rautio $\mathrm{M}$, Jousimies-Somer $\mathrm{H}$, Kauma $\mathrm{H}$, et al. Liver abscess due to a lactobacillus rhamnosus strain indistinguishable from $\mathrm{L}$. rhamnosus strain GG. Clin Infect Dis. 1999;28:1159-60.

32. Gueimonde M, Sánchez B,G, de los Reyes-Gavilán C, et al. Antibiotic resistance in probiotic bacteria. Front Microbiol. 2013:4:202

33. Salminen MK, Rautelin H, Tynkkynen S, et al. Lactobacillus bacteremia, species identification, and antimicrobial susceptibility of 85 blood isolates. Clin Infect Dis. 2006:42:e35-44

34. Musso M, Capone A, Chinello P, et al. Extra-cerebral severe infections associated with haemorrhagic hereditary telangiectasia (Rendu-Oslerweber disease): five cases and a review of the literature. Infez Med. 2014:22:50-6.

35. Duval X, Djendli S, Le Moing V, et al. Recurrent Staphylococcus Aureus extracerebral infections complicating hereditary hemorrhagic telangiectasia (Osler-Rendu-weber disease). Am J Med. 2001;110:671-2.

36. Hull HF, Mann JM, Sands CJ, et al. Toxic shock syndrome related to nasal packing. Arch Otolaryngol. 1983;109:624-6.

37. Shovlin C, Bamford K, Wray D. Post-NICE 2008: antibiotic prophylaxis prior to dental procedures for patients with pulmonary arteriovenous malformations (PAVMs) and hereditary haemorrhagic telangiectasia. Br Dent J. 2008:205:531-3.

38. Van Rijen M, Bonten M, Wenzel R, et al. Mupirocin ointment for preventing Staphylococcus Aureus infections in nasal carriers. Cochrane Database Syst Rev. 2008; https://doi.org/10.1002/14651858.CD006216.pub2. 